\title{
Optical behavior of surface bubbles
}

\author{
Samuele Straulino, Cecilia M C Gambi and \\ Giuseppe Molesini
}

Department of Physics and Astronomy, University of Florence, via G. Sansone 1, 50019 Sesto Fiorentino, Italy

E-mail: samuele.straulino@unifi.it

Received 1 September 2015

Accepted for publication 11 September 2015

Published 6 October 2015

\begin{abstract}
The observation of diamond-like light spots produced by surface bubbles obliquely illuminated is reported. The phenomenon is discussed in terms of geometrical optics, and an explanation is provided attributing the effect to the astigmatism introduced by the deformation of the liquid surface surrounding the bubble. An essential ray tracing program is outlined and used to reconstruct the observed phenomenon numerically.
\end{abstract}

Keywords: refraction, ray tracing, soap bubble

(Some figures may appear in colour only in the online journal)

\section{Introduction}

Soap bubbles are among the most fascinating subjects of investigation, involving physics of liquids, basic matter interactions, and optical properties of thin films [1-3]; specific measuring techniques have been developed to study their formation and lifetime behavior [4-6]. In addition, visual observation of the beautiful colors of floating soap bubbles offers an excellent opportunity to discuss basic interference phenomena at the educational level.

Another opportunity to approach optics concepts such as refraction and image formation is given by surface bubbles. These are usually observed in everyday life, whenever some inner gas emerges to the surface of a liquid mass, or some air is mixed to a liquid because of gurgling or turbulent flow. Such bubbles may last a few seconds and then burst, or may survive for a longer time, as for example with surface bubbles obtained from an aqueous solution of a surfactant material. In the presence of a bubble, the liquid surface is deformed; moreover, considering a basin such as a pan or a kitchen sink with water in, if the bubble is illuminated by a far source or an almost collimated light beam, at the bottom it is common to see a light spot whose features may be various, depending on the illumination angle. In particular, surface bubbles with a diameter of the order of $1 \mathrm{~cm}$ and water depth about $10 \mathrm{~cm}$, 


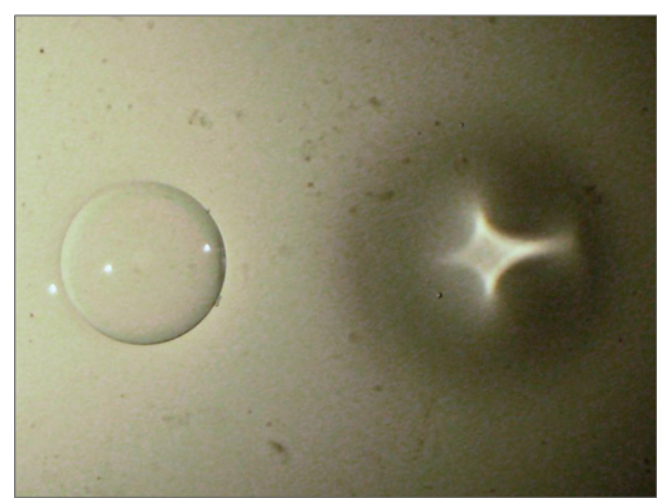

Figure 1. Soap bubble at the surface of a pan with water, and light spot projected at the bottom. The bubble diameter is $17 \mathrm{~mm}$.

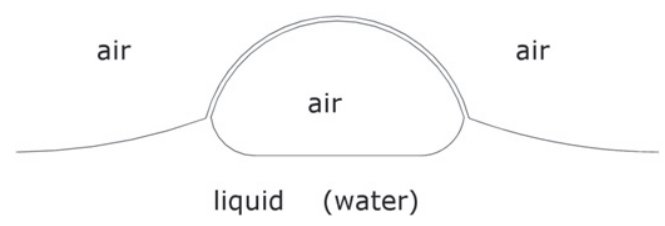

Figure 2. Schematic profile of a surface soap bubble.

obliquely illuminated with a light beam at $30^{\circ}$ or more to the normal, are seen to produce a diamond-like light spot similar to that shown in figure 1 . The phenomenon is recurring and at all reproducible, but so far an explanation does not appear to have been given. Here we present a treatment based on geometrical optics, accounting for the mechanisms that are responsible for the reported features. The approach moves from the simple observation of surface bubbles in everyday life, replicates the relevant issues in the laboratory, figures out a way of explanation, studies a model accordingly, develops the computational tools for verification, and checks the outcomes with respect to the experimental observation. The educational content is adequate for undergraduate students with an interest in optics and for computer simulation of physics phenomena. Along the line of similar activities [7-9], a potential application for the topic is within a physics project lab, where it can be proposed as an exercise aimed at strengthening the investigative skills and analysis capacity towards applied research.

\section{Profile of a surface bubble}

The profile of a surface bubble is qualitatively shown in figure 2 [10]. Such a profile can be distinguished in three main parts: the upper cup, the bottom hollow, and the outer slope.

The upper cup is made of a thin film, of the same structure as floating soap bubbles. Its thickness may range from a few nanometers to a few microns [11-15]. Using as a model a pair of concentric spherical shells of inner radius $R$ and outer radius $R+\mathrm{d} R$ with a thin water layer in between, the upper cup constitutes a lens, whose focal length $f$ can be obtained from the lensmaker's equation [16]: 


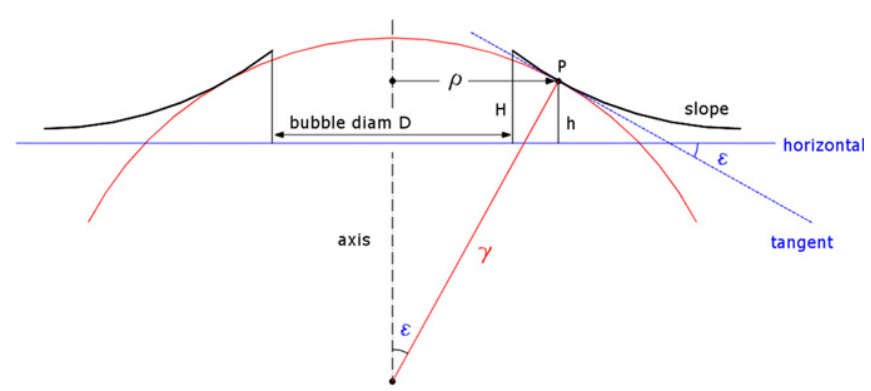

Figure 3. Assimilating the outer slope of the bubble at a point $P$ to a lens surface of radius $\gamma$.

$$
\frac{1}{f}=(n-1)\left(\frac{1}{R+\mathrm{d} R}-\frac{1}{R}\right) \simeq-(n-1) \frac{\mathrm{d} R}{R^{2}},
$$

where it is assumed that $\mathrm{d} R \ll R$, as is the case with soap bubbles of common size; $n$ is the refractive index of the water layer $(n=1.33)$. After equation (1) it is seen that the cup behaves as a diverging lens of very long focal length, and therefore it is unable to account for the light spot in figure 1 .

The bottom hollow of the surface bubble is almost flat, with a concave profile at the edge. The refracted light is either just deflected at a fixed angle (zero at normal incidence) by the flat portion of the surface, or outward directed by the concave edge. Neither portion, therefore, can account for the observed effect of light concentration.

The outer slope behaves instead as a focusing device. With reference to figure 3 , a ray impinging on the slope at a point $P$ is inward refracted, as if it were impinging on a spherical surface of radius $\gamma$ given by

$$
\gamma=\frac{\rho}{\sin \varepsilon}
$$

where $\rho$ is the radial distance of $P$ from the bubble axis, and $\varepsilon$ is the angle between the tangent to the surface in $P$ and the horizontal line. As far as refraction is concerned, the slope can therefore be assimilated to a continuous set of spherical lenses whose radius of curvature is given by equation (2) and whose center lies along the bubble axis. The mathematical expression for $\varepsilon(\rho)$ depends on the actual physics parameters describing the structure of the surface bubble; for the purpose of this work, we refer to a heuristic model where the slope height $h$ is a negative exponential of the form

$$
h(\rho)=H \mathrm{e}^{-\alpha(\rho-D / 2)},
$$

with $D$ the bubble diameter, $H=h(D / 2)$, and $\alpha$ the exponential decay constant, so that

$$
\varepsilon(\rho)=\arctan \frac{\partial h}{\partial \rho}=-\arctan \left[\alpha H \mathrm{e}^{-\alpha(\rho-D / 2)}\right] .
$$

The overall situation for a collimated beam of rays parallel to the bubble axis is qualitatively depicted in figure 4 . To proceed further, and provide an explanation for the diamondlike light spot in figure 1 , it is necessary to trace a set of rays through the air-water interface given by equation (3). 


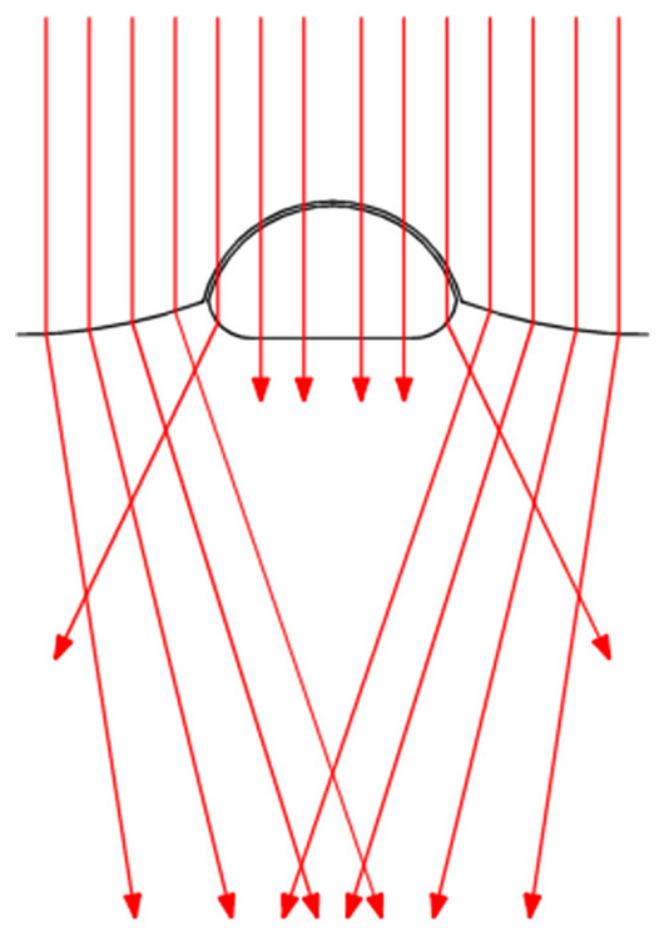

Figure 4. Focusing effect of a surface bubble on a collimated beam of rays parallel to the bubble axis.

\section{Tracing rays through the outer slope of a surface bubble}

When passing through an interface, a light ray is bent according to the refraction law

$$
n \sin i=n^{\prime} \sin i^{\prime},
$$

with $n$ the refractive index of the first medium, $i$ the angle of incidence, $n^{\prime}$ the refractive index of the second medium, and $i^{\prime}$ the refraction angle. The task of ray tracing is relatively simple for rays that belong to a plane containing the lens axis (meridional rays), since the surface normal also belongs to the same plane, as well as the refracted ray. For all other rays (skew rays) it is necessary to consider the refraction law in its vector form; with clarifications as given in recent literature [17], such a law is written

$$
n \hat{r} \times \hat{a}=n^{\prime} \widehat{r^{\prime}} \times \hat{a},
$$

where $\hat{r}$ is the unit vector singling out the incident ray, $\hat{a}$ the unit vector of the surface normal, and $\widehat{r^{\prime}}$ the unit vector of the refracted ray. The Cartesian components of such unit vectors are given by the respective direction cosines. For meridional rays, equation (6) directly reduces to equation (5); for skew rays, equation (6) also expresses the belonging of the refracted ray $\widehat{r^{\prime}}$ to the incidence plane defined by $\hat{r}$ and $\hat{a}$.

Converting equation (6) to a set of algebraic equations and solving for $\widehat{r^{\prime}}$ implies a simple but somewhat lengthy computation, and is here deferred to the literature [18-23]. Besides, there are several commercially available programs that provide appropriate means to trace rays through optical systems in general, allowing to deal with a number of lenses, mirrors, diffractive optics and more. Such programs, however, are typically conceived as an aid to 
optical design, and require a specific training for their use. On the other hand, our refractive surface (the outer slope of the bubble) has a peculiar profile, quite different from that of common lenses. We then wrote a simple computer routine on the basis of equation (6), providing the data of the refracted ray after an assigned incidence ray, and also propagating the refracted ray to a given image plane. Tracing a set of incidence rays, the spot diagram of the locations where the refracted rays pierce the image plane is obtained. Properly selecting the set of incidence rays, such a spot diagram is indicative of the light spot that is expected on a screen after refraction from the outer slope of the surface bubble. The conditions that determine the spot shape of figure 1 are then investigated.

As to our essential ray tracing program, it is made of a main section and a ray tracing routine. In the main section, a set of data points on the refracting surface is selected; such points are singled out by means of their $(x, y, z)$ coordinates. At these locations, the direction cosines defining the unit vector $\hat{a}$ of the surface normal are computed making use of equation (3). The direction cosines defining the incident ray $\hat{r}$ are given by assigning the field angle; the latter direction cosines are the same for the entire set of data points. Also assigned is the axial distance from the refracting surface to the image plane. At each data point of the set, the main program makes a call to the ray tracing routine, providing as input data the $(x, y$, $z$ ) coordinates of the point, the direction cosines of the local surface normal, those of the incident ray, and the distance to the image plane. The ray tracing routine performs the computations implied by equation (6) (we use the mathematics detailed in reference [19] ) and works out the direction cosines of the refracted ray $\widehat{r^{\prime}}$. Such a ray is then propagated to the image plane, providing as output data the $(x, y)$ coordinates of the point where $\widehat{r^{\prime}}$ intersects the image plane. Ray by ray, the main program collects all such output points in a file, to be plotted as a spot diagram by external graphics programs.

Although only limited to meridional rays, validation of our routine was obtained by comparing our outcomes with the results of single ray tracing from equation (5). In that case the sequence of computations is much simpler, and does not imply the explicit use of direction cosines $[24,25]$. Coincidence of the results was obtained up to the last significant digit.

\section{Generation of astigmatic spot diagrams}

Thinking of the spot diagram as the result of refraction on a continuous set of spherical lenses axially displaced, aberrations need to be considered. In particular, depending upon the square of the field angle, astigmatism is expected to become dominant when the illuminating beam impinges on the bubble at large angle. Astigmatism is known to produce two focal lines, one named meridional (or tangential) line, due to rays belonging to the meridional plane, and the other named sagittal line, due to rays on the sagittal plane; the latter contains the chief ray and is normal to the meridional plane (figure 5).

The lenses of the set making up the outer slope of the surface bubble have different focal lengths, so that at a fixed distance from the bubble where the screen is located it is possible to have either the meridional line or the sagittal line (or intermediate or external spots), according to the particular lens of the set that is considered. For all the lenses of the set together, all such lines and spots will be superimposed. It is therefore conceivable that the actual light distribution be made of a basic diamond-like spot, with the meridional and sagittal focal lines as diagonals.

To simulate the behavior of a surface bubble with our model and ray tracing program, we set the bubble diameter to $D=10 \mathrm{~mm}$, and selected a guess value of the parameters of the 


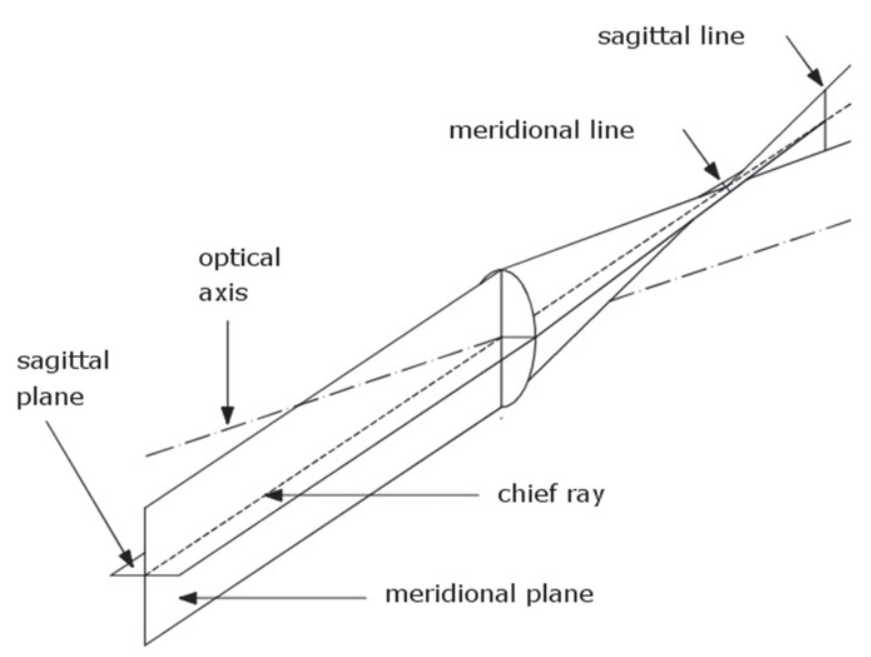

Figure 5. Meridional and sagittal lines produced by a lens because of astigmatism.

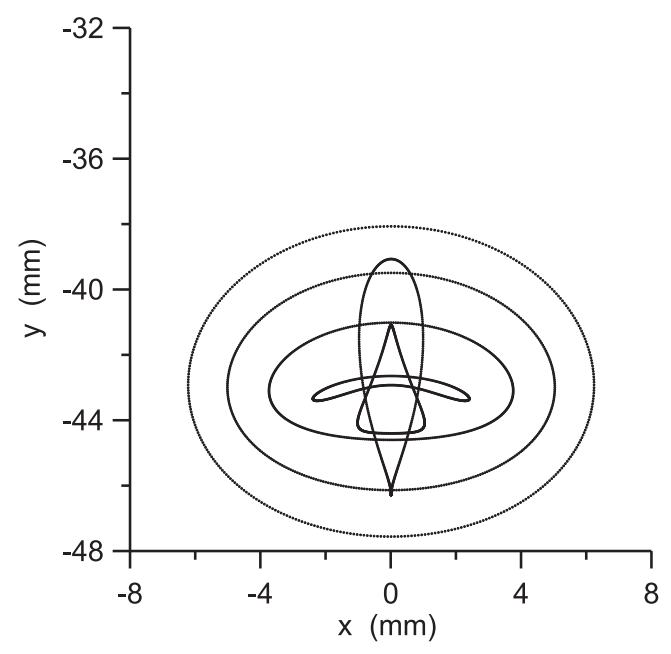

Figure 6. Spot diagram calculated from a series of six crowns of rays about the surface bubble.

negative exponential to $H=2 \mathrm{~mm}$ and $\alpha=0.2 \mathrm{~mm}^{-1}$. The selected illumination angle was $45^{\circ}$. We then considered a series of incident rays impinging on a circle laying on the slope at an azimuthal separation of $1^{\circ}$, namely, a circular crown of 360 rays around the surface bubble. Referring to a distance of $70 \mathrm{~mm}$ (found by trial) from the bubble, and to six equally spaced ray crowns from $\rho_{\min }=D / 2+1 /(3 \alpha)$ to $\rho_{\max }=D / 2+1 / \alpha$, the resulting spot diagram is shown in figure 6.

As it appears, the shape of the crowns, initially circular, is variously deformed in a manner that is reminiscent of the meridional and sagittal lines due to astigmatism (the narrow lines drawn in figure 5 relate to a first approximation, and omit the contribution of other aberrations). To draw the expected shape of the light spot as a whole, we considered a more 


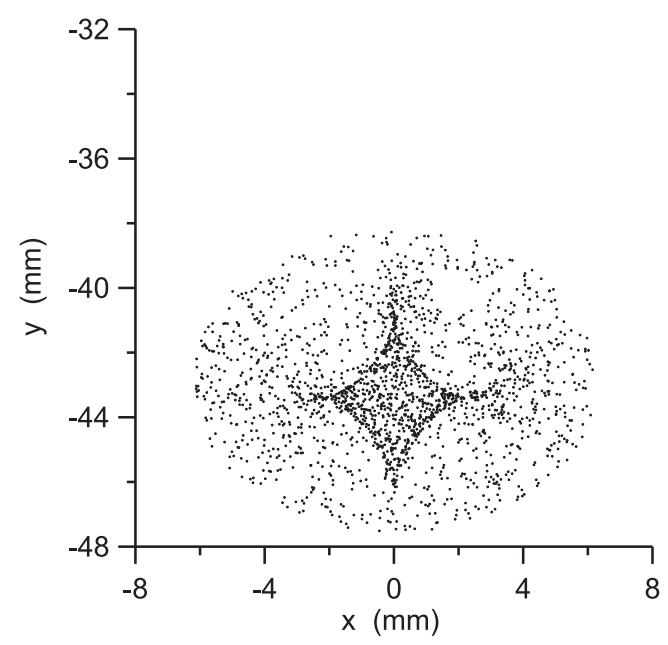

Figure 7. Spot diagram calculated from a random set of rays within an annular ring about the surface bubble.

Table 1. Values of the parameters in equation (7) and offset $Z_{0}$ for verification.

\begin{tabular}{|c|c|}
\hline Parameter & Numerical value \\
\hline$c v$ & $4.87210^{-2}$ \\
\hline$c c$ & $-2.26310^{-1}$ \\
\hline$a d$ & $1.12210^{-5}$ \\
\hline$a e$ & $-8.43610^{-7}$ \\
\hline af & $-1.00710^{-8}$ \\
\hline$a g$ & $9.05610^{-11}$ \\
\hline$Z_{0}$ & $-4.58210^{-1}$ \\
\hline
\end{tabular}

general set of rays, filling up the annular ring between the same $\rho_{\min }$ and $\rho_{\max }$ with a set of rays at random locations. The result is shown in figure 7 , where the similarity with the spot of figure 1 is evident.

For an independent check of our computations, we also approached the ray tracing problem with OSLO-EDU, a standard program commonly used for teaching purposes [26]. Referring at first to a spherical refracting surface, using the single ray trace option of the program on a choice of skew rays we further verified the validity of our ray tracing routine. Coming then to surface bubbles, a major difficulty that we encountered was given by the peculiar shape of the refracting surface - a negative exponential — which greatly differs from a definite sphere. To represent our surface we used the expression of the sag $z(\rho)$ in the case of general aspherics

$$
z(\rho)=\frac{c v \rho^{2}}{1+\sqrt{1-c v^{2}(c c+1) \rho^{2}}}+a d \rho^{4}+a e \rho^{6}+a f \rho^{8}+a g \rho^{10}
$$

with $c v$ the curvature (reciprocal of the radius of curvature) of the paraxial sphere, $c c$ the conic constant ( $c c>0$ for an oblate spheroid, $c c=0$ for a sphere, $-1<c c<0$ for an ellipsoid, $c c=-1$ for a paraboloid, $c c<-1$ for a hyperboloid), and $a d$, $a e$, af, ag the coefficients of a radial power expansion. The above sag $z(\rho)$ should be related to the sag $Z(\rho)$ produced by the 


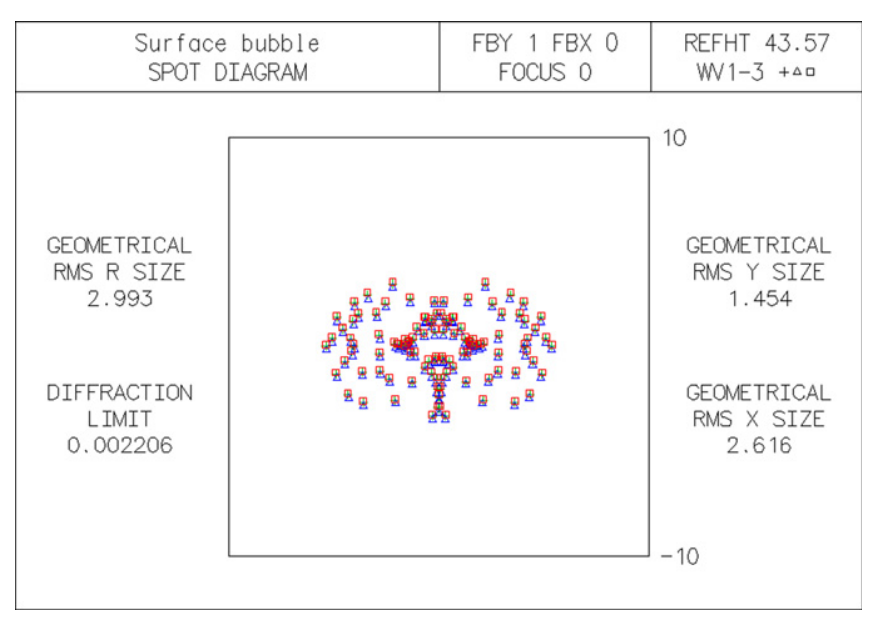

Figure 8. Spot diagram obtained with the program OSLO-EDU under conditions similar to those of figure 7. Symbols refer to different wavelengths: crosses to $588 \mathrm{~nm}$, triangles to $486 \mathrm{~nm}$, squares to $656 \mathrm{~nm}$.

negative exponential when the reference height is taken at $\rho=\mathrm{D} / 2$, so that

$$
Z(\rho)=Z_{0}+H\left[1-\mathrm{e}^{-\alpha(\rho-D / 2)}\right],
$$

with $Z_{0}$ a constant offset to be determined. To have $z(\rho) \approx Z(\rho)$ in the radial range between $\rho_{\min }$ and $\rho_{\max }$, it is found that the values for the parameters appearing in equation (7) and for the offset may be given as in table 1. Using such values, the aspheric sag differs from that given by the negative exponential by less than $1 \%$. Such an aspheric surface was introduced in OSLO-EDU as an air-water interface, also adding a central obstruction of radius $\rho_{\min }$ followed by a transmissive opening of radius $\rho_{\max }$. At $45^{\circ}$ field angle, the resulting spot diagram is shown in figure 8 , where symbols of different shape and color are used for standard wavelengths $588 \mathrm{~nm}, 486 \mathrm{~nm}, 656 \mathrm{~nm}$, customarily referred to in optical design. Comparing these results with the spot diagram of figure 7 , it is seen that the outcomes are in fair agreement, qualitatively accounting for the diamond-like spot of figure 1 .

\section{Conclusions}

Starting from a common observation of diamond-like light spots produced by surface bubbles, an explanation in terms of geometrical optics has been provided. The phenomenon is interpreted as an effect mainly due to astigmatism of the aspheric surface surrounding the bubble, described as a continuous set of osculating spheres; ray tracing, carried out with an essential program, does confirm the analysis and also provides a tool to adjust the parameters of the surface profile so that the experimental observation is better accounted. Proceeding further, the approach described here could be used as a method for measuring the surface deformation of a liquid surface under various conditions, attracted for example by the illustrations of light distributions due to surface ripples and water drops reported in the literature [27].

As to educational content, the exercise we presented is useful to apply to a practical case the notion of optical aberrations, and to approach the basic methods of ray tracing. The task is particularly appropriate to a physics project lab, envisaging a working path that develops along the same basic lines as applied research. 


\section{Acknowledgments}

The authors wish to sincerely thank Marcello Colocci, who made the early observations of diamond-like light spots produced by surface bubbles, and inspired our work to provide a physical explanation of the phenomenon. We are also grateful to Annamaria Cartacci for encouragement and useful comments, and to Marcello Carlà for thorough and fruitful discussions on the subject of surface soap bubbles.

\section{References}

[1] Boys C V 1958 Soap Bubbles and the Forces that Mould Them (New York: Dover)

[2] Mysels K J, Shinoda K and Frankel S 1959 Soap Films, Studies of Their Thinning (New York: Pergamon)

[3] Isenberg C 1992 The Science of Soap Films and Soap Bubbles (New York: Dover)

[4] Afanasyev Y D, Andrews G T and Deacon C G 2011 Measuring soap bubble thickness with color matching Am. J. Phys. 79 1079-82

[5] Lv W, Zhou H, Lou C and Zhu J 2012 Spatial and temporal film thickness measurement of a soap bubble based on large lateral shearing displacement interferometry Appl. Opt. 51 8863-72

[6] Vannoni M, Sordini A, Gabrieli R, Melozzi M and Molesini G 2013 Measuring the thickness of soap bubbles with phase-shift interferometry Opt. Express 21 19657-67

[7] Molesini G and Vannoni M 2008 Light reflection in a pool under falling rain droplets Eur. J. Phys. 29 403-11

[8] Molesini G and Vannoni M 2009 Light reflection from water surfaces perturbed by falling rain droplets Eur. J. Phys. 30 1009-14

[9] Gluck P and King J 2015 Physics Project Lab (Oxford: Oxford University Press)

[10] Debrégeas G, de Gennes P-G and Brochard-Wyart F 1998 The life and death of 'bare' viscous bubbles Science 279 1704-7

[11] Bélorgey O and Benattar J J 1991 Structural properties of soap black films investigated by x-ray reflectivity Phys. Rev. Lett. 66 313-6

[12] Lionti-Addad S and Di Meglio J M 1992 Stabilization of aqueous foam by hydrosoluble polymers: 1. Sodium dodecyl sulphate-poly(ethylene oxide) system Langmuir $8324-7$

[13] Greco V, Iemmi C, Ledesma S, Molesini G, Puccioni G P and Quercioli F 1994 Measuring soap black films by phase shift interferometry Meas. Sci. Technol. 5 900-3

[14] Greco V and Molesini G 1996 Monitoring the thickness of soap films by polarization homodyne interferometry Meas. Sci. Technol. 7 96-101

[15] Gambi C M C, Vannoni M, Sordini A and Molesini G 2014 Monitoring the thinning dynamics of soap films by phase shift interferometry. The case of perfluoropolyether surfactants Eur. Phys. J. E 37 1-8

[16] Hecht E 1987 Optics 2nd edn (Reading, MA: Addison-Wesley) p 138

[17] Tkaczyk E R 2012 Vectorial laws of refraction and reflection using the cross product and the dot product Opt. Lett. 37 972-4

[18] Kidger M 2002 Fundamental Optical Design (Bellingham, WA: SPIE) pp 48-54

[19] Smith W J 1966 Lens Design Fundamentals (New York: McGraw-Hill) pp 254-62

[20] Welford W T 1974 Aberrations of the Symmetrical Optical System (London: Academic) pp 47-53

[21] Born M and Wolf E 19755th edn. Principles of Optics (Oxford: Pergamon) pp 194-6

[22] Kingslake R 1978 Lens Design Fundamentals (New York: Academic) pp 145-50

[23] Malacara D and Malacara Z 2004 Handbook of Optical Design (New York: Dekker) appendix 4

[24] Smith G and Atchison D A 1997 The Eye and Visual Optical Instruments (Cambridge: Cambridge University Press)

[25] Molesini G 2005 Geometrical optics Encyclopedia of Condensed Matter Physics ed G Bassani, G Liedl and P Wyder (Oxford: Elsevier) pp 257-67

[26] www.lambdares.com/oslo-edu

[27] Nye J F 1999 Natural Focusing and Fine Structure of Light: Caustics and Wave Dislocations (Bristol: Institute of Physics Publishing) 